

\title{
Gestión Estratégica, Crecimiento Económico y Productividad
}

Manuel A. Muñoz Suárez Compilador 
Ediciones UTMACH

226 pág: $21 \times 29,7 \mathrm{~cm}$

Colección Monográficos

Título: Gestión estratégica, crecimiento económico y productividad - Manuel A. Muñoz Suárez (Compilador)

Primera edición 2020

ISBN: 978-9942-24-138-2

CDD 330

1. Economía,

2. Producción

--Temas relacionados

Publicación PDF 
Gestión estratégica, crecimiento económico y productividad

\author{
Manuel A. Muñoz Suárez
}

COMPILADOR

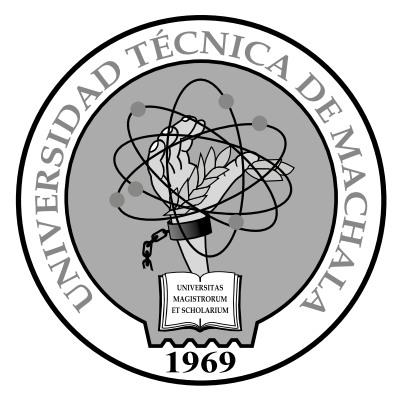


Autoridades

César Quezada Abad - Rector

Amarilis Borja Herrera - Vicerrector Académico

Jhonny Pérez Rodríguez - Vicerrector Administrativo

Luis Brito Gaona

Director de Investigación

( ) Ediciones UTMACH

Colección Monográficos

Título original:

Getsión estratégica, crecimiento económico y productividad

ISBN: 978-9942-24-138-2

(c) Manuel Muñoz Suárez

(Compilador)

(c) Autores de capítulos

DOI: http://doi.org/10.48190/9789942241382

Primera edición 2020

Karina Lozano Zambrano

Jefe editor / Diseño y edición editorial

Fernanda Tusa Jumbo - Corrector de estilos Jorge Maza-Cordova - Asesor tecnológico

Karla Ibañez y Cyndi Aguilar - Equipo de difusión

Este obra está bajo una licencia de Creative Commons Reconocimiento No Comercial.

Esta licencia permite a otros entremezclar, ajustar y construir a partir de su obra con fines no comerciales, siempre y cuando le reconozcan la autoría y sus nuevas creaciones estén bajo una licencia con los mismos términos. 


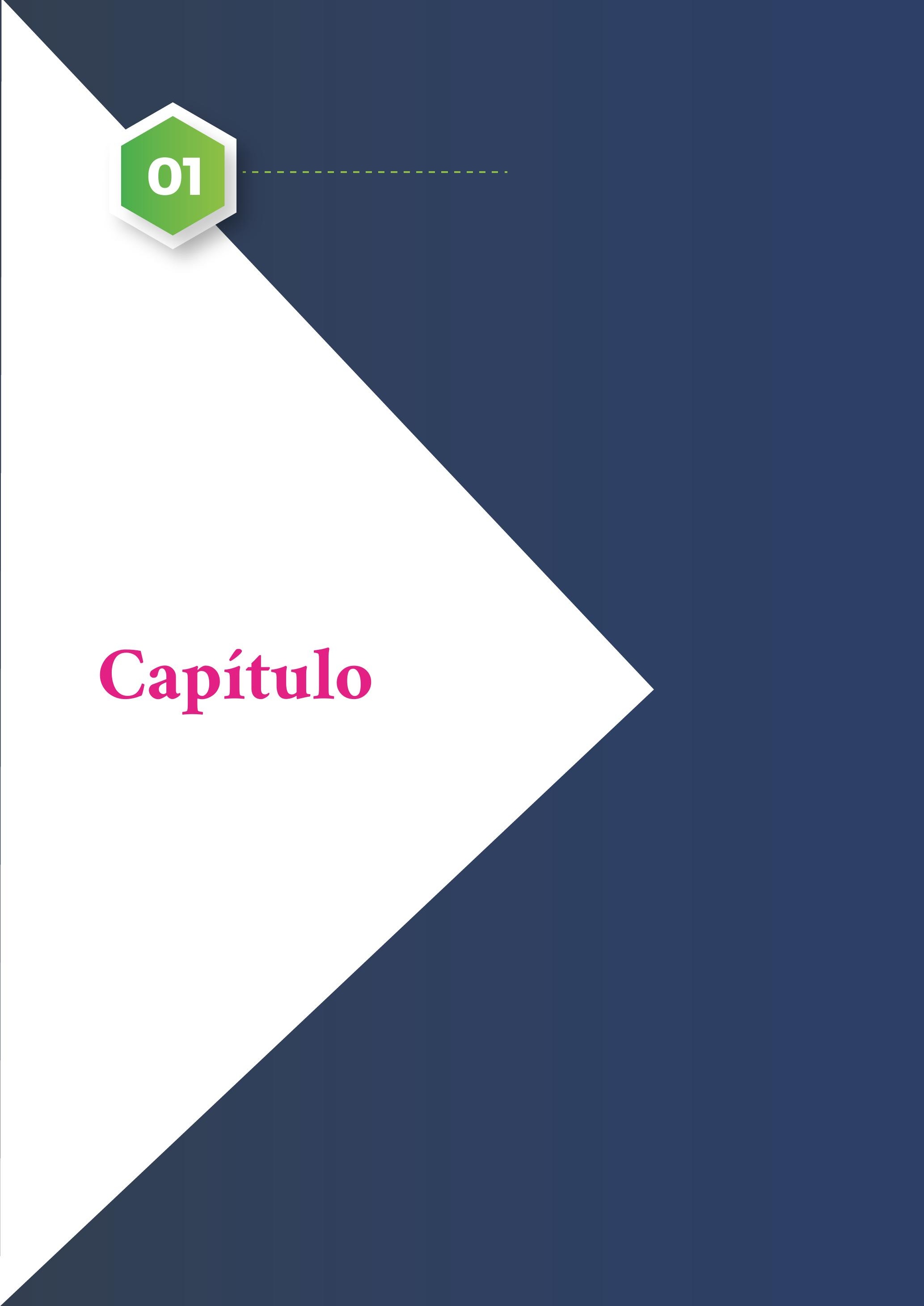


Crecimiento económico: opciones de política económica

\author{
Flor Yelena Vega Jaramillo \\ Jorge Guido Sotomayor Pereira \\ Lenny Beatriz Capa Benítez
}

Autores 


\section{Crecimiento económico: opciones de política económica}

\section{Flor Yelena Vega Jaramillo}

Doctoranda en Ciencias Económicas en la Universidad del Zulia - Venezuela.

Docente titular de la Universidad Técnica de Machala, Ecuador.

\section{Jorge Guido Sotomayor Pereira}

Doctor en Análisis Económico y Estrategia Empresarial por la Universidad de la Coruña, España. Docente titular de la Universidad Técnica de Machala.

\section{Lenny Beatriz Capa Benítez}

Doctora en Ciencias Administrativas por la Universidad Nacional Mayor de San Marcos. Docente de la Universidad Técnica de Machala.

DOI: http://doi.org/10.48190/9789942241382.1 


\section{Resumen}

El crecimiento económico es un objetivo fundamental de los países, se considera que al aumentar la riqueza total de una nación, también se ven mejoradas las posibilidades de reducir la pobreza. Su análisis se ha convertido en una preocupación constante de las ciencias económicas, estudiándolo desde diferentes enfoques y teorías. Dentro de este contexto es importante resaltar el papel del gobierno, requiriéndose además, de la participación público privada para identificar las necesidades de políticas públicas para de esta manera lograr un aumento de la productividad. El presente trabajo tiene por objetivo analizar el crecimiento económico basado en las decisiones de política económica tomadas por el gobierno de Ecuador. De esta manera los objetivos específicos son: a) analizar la política económica del país, antes y después de la dolarización; b) la política fiscal y el crecimiento económico de Ecuador; c) analizar la relación entre gasto público y el crecimiento económico; d) determinar la importancia de la inversión pública en el crecimiento económico. La metodología de la investigación responde a una revisión bibliográfica, lo que permitió iniciar la búsqueda de la información en diferentes fuentes: normas, reportes oficiales, revistas especializadas, memorias de conferencias, tesis doctorales, entre otros. Se concluye que los pilares del crecimiento económico de Ecuador a partir del año 2000, fue la aplicación de una política fiscal, que validaba el aumento del gasto público, el incremento de la recaudación tributaria, y las transferencias sociales. Esto ha promovido el déficit fiscal, es decir al gasto excesivo de los recursos del Estado.

Palabras claves: crecimiento económico, política económica, política fiscal, política monetaria, dolarización

\section{Introducción}

Los ciudadanos son testigos de cómo un país, a pesar de los gobiernos de turno, mantienen sus políticas económicas guiadas por los objetivos de bienestar para la población, lo que incluye principalmente el incremento del bienestar social a partir de una mayor producción, inversión, empleo y equidad social. Estás políticas, de acuerdo a García y Zabala (2008) se conciben como una totalidad coherente y conexa de valores éticos - políticos, de esta manera su identidad material recae en el conjunto de valores supremos y principios fundamentales que las caracterizan de cualquier norma legal, la aplicación de políticas económicas buscan el bienestar de la sociedad, lográndose a través del crecimiento y el desarrollo económico.

El crecimiento económico es un objetivo fundamental de los países, porque se considera que al aumentar la riqueza total de una nación, también se ven mejoradas 
las posibilidades de reducir la pobreza y resolver los problemas sociales de la población. El análisis del crecimiento económico, el cual se entiende como el resultado de la dinámica entre las formas de producción, relaciones de poder e instituciones de desarrollo, se han convertido en una preocupación constante de la ciencia económica, que ofrece su análisis desde diferentes enfoques y teorías.

Existen muchas maneras o puntos de vista a partir de los cuales se puede medir el crecimiento económico que presenta una sociedad, entre las variables que contribuyen a esta medición se encuentran: la inversión, las tasas de interés, el nivel de consumo, las políticas gubernamentales o la tasa de ahorro. Las variables mencionadas son herramientas que les permiten a los países establecer que tan cerca o lejos se encuentran del desarrollo. Los principales objetivos de las políticas económicas son: a) lograr un crecimiento económico sostenido en el tiempo; b) conseguir la estabilidad de los precios; y, c) favorecer el empleo. Mientras que las principales políticas económicas que aplican los estados son: Política Monetaria, Política Fiscal, Política exterior y Política de Rentas.

Por lo tanto, la importancia del estudio del crecimiento económico recae principalmente en la relación con otras variables macroeconómicas, con respecto a la conducción de las políticas económicas y por su impacto sobre el bienestar presente y futuro de la población. El que los Estados diseñen una política que sea adecuada para lograr un determinado objetivo, es sin duda uno de los análisis económicos con mayor transcendencia en la actualidad. Este análisis es considerado de gran complejidad, debido a que es necesario establecer las relaciones que existen entre los instrumentos que se encuentran disponibles, así como la magnitud que presentaran sus efectos sobre el objetivo escogido, de esta manera los Estados podrán elegir los instrumentos idóneos y la intensidad con la que podrán ser utilizados, por lo que la elección del objetivo resulta de gran importancia.

De acuerdo a lo expuesto, en el presente capítulo se estudiará a la política económica, la misma que es revisión de los diferentes instrumentos de materia fiscal, monetaria y cambiaria, donde los protagonistas y hacedores de la política son el poder Ejecutivo, Legislativo, Banco Central, entre otros, los cuales tratan de ayudar para que los mercados generen equilibrio, donde sus principales propósitos son: el crecimiento y la estabilidad económica. El objetivo general del capítulo es analizar el crecimiento económico basado en las decisiones de política económica tomadas por el gobierno de Ecuador.

\section{Marco conceptual}

Antes de analizar la política económica de Ecuador, es necesario definir qué es política económica y su importancia para lograr el crecimiento de los Estados. Se sabe que los agentes económicos como lo son: familia, empresa y Estado se encargan 
de realizar los procesos encaminados hacia la obtención de los bienes y servicios que son necesarios para cubrir las necesidades de la sociedad donde se encuentran, y dentro de ese contexto es el Estado el encargado de regular las actividades económicas con el propósito de obtener el máximo bienestar económico. También se conoce que el mercado no funciona de una manera totalmente eficaz, por lo que puede presentar fallas en la asignación de los recursos disponibles y deficiencias en lo que respecta a la distribución de la renta, razón por la cual se exige la intervención del Estado para corregir estos fallos o al menos tratar de disminuir su impacto. En la Figura 1 se presentan los posibles fallos de mercado y la función que tendría el Estado para mitigarlos.

Figura 1. Funciones del Estado frente a las fallas del mercado

\begin{tabular}{|l|l|l|}
\hline \multicolumn{2}{|c|}{ Fallo del mercado } & \multicolumn{1}{c|}{ Función del Estado } \\
\hline $\begin{array}{l}\text { No logra controlar la inestabilidad } \\
\text { que presentan los ciclos económicos. }\end{array}$ & Estabilizar la economía \\
\hline $\begin{array}{l}\text { No garantiza la satisfacción de las ne- } \\
\text { cesidades colectivas más básicas. }\end{array}$ & $\begin{array}{l}\text { Suministrar bienes y servicios } \\
\text { públicos }\end{array}$ & \\
\hline $\begin{array}{l}\text { No pone en consideración las exter- } \\
\text { nalidades como los costos y beneficios } \\
\text { externos. }\end{array}$ & Regular el medio ambiente. \\
\hline $\begin{array}{l}\text { Posibilita la falta de competencia. } \\
\text { Genera una distribución desigual de } \\
\text { la renta }\end{array}$ & Redistribuir la renta. \\
\hline
\end{tabular}

Nota: Política Económica, crecimiento y desarrollo humano: principales relaciones. Reyes (2008).

De esta manera surgen las diferentes funciones del Estado en la economía, cuya ejecución y desarrollo dan paso a la política económica. Por lo tanto se conoce como Política Económica al conjunto de normas y decisiones a través de las cuales el Estado se encarga de regular y al mismo tiempo brindar orientación sobre aquellas actividades que se realizan dentro del país. Teniendo en cuenta que el propósito que tiene cualquier economía es la de lograr el crecimiento. Considerando el equilibrio de los mercados la política económica debe dirigirse a explicar y enfrentar fenómenos económicos como lo son la inflación, el desempleo, la devaluación y todo aquello que signifique llevar a la estabilización de los precios (Carrillo, 2017).

Lograr el crecimiento económico, implican que los gobiernos tomen decisiones importantes dentro del ámbito de la política pública. De esta manera tomando como base el modelo neoclásico, la política debería principalmente concentrarse en el ahorro y la inversión; por otra parte el modelo dinámico de competencia implica que la política debe favorecer la promoción de la libertad económica (Hunt, 2007). 
En la actualidad, es imprescindible que se realicen amplias concertaciones nacionales en áreas identificadas como de gran prioridad como lo son la lucha contra la pobreza, la educación, la salud, el microcrédito hacia los emprendedores, la disminución del desempleo, el apoyo a la pequeña y mediana empresa (pymes) y todas aquellas que en realidad impliquen democratizar las oportunidades productivas (Rodríguez \& Rodríguez, 2009).

Para Valdivieso (2015) la aplicación de la política económica compromete al gobierno a buscar el contexto adecuado, donde se pueda hacer uso eficiente de las capacidades productivas que presenta el país, llegando a optimizar los niveles de productividad y a lograr el desarrollo económico y social que buscan ofrecer un mejor nivel de vida a la población en general. Por lo tanto, a partir de la aplicación de la política económica las autoridades esperan lograr un mayor control sobre la economía de los países, esperando lograr, como ya se mencionó anteriormente, estabilidad y crecimiento económico.

De acuerdo a lo expresado, se considera que la política económica va más allá de lo que significa la teoría económica, la teoría económica transita entre la economía normativa y la economía positiva. La economía positiva se basa en especificar, demostrar y responder a situaciones, desde un punto de vista objetivo; mientras que la economía normativa busca dar opiniones personales de manera subjetiva. Por lo tanto no solo se explica porque suceden los fenómenos económicos, sino que ofrece direccionamientos para poder utilizar de forma eficiente los recursos, explicando también el impacto de estas políticas en la sociedad (Cachanosky, 2012). En este aspecto, tanto los fines como los medios están supeditados, en gran medida a la ideología (Gutiérrez, 2009). Es decir, la política económica se preocupa principalmente de aplicar aquellas teorías que son elaboradas desde la teoría económica, en otras palabras, no solo investiga las causas por las que suceden los hechos, sino que también provee de las herramientas que son necesarias para superar los conflictos o potenciar las oportunidades que se presenten.

También es indispensable mencionar que para llevar a cabo una adecuada aplicación de la política económica, es necesario poner en consideración los comportamientos que presentan las variables a nivel nacional, así como también disponer de elementos que permitan su adecuada implementación (Torres \& Rojas, 2015). Para llevar a cabo lo mencionado, el Estado dispone de un conjunto de elementos, provenientes de la política fiscal, monetaria y cambiaria (Figura 2), a través de ellos puede delegar ciertas acciones, donde su correcta aplicación también depende, en gran medida, del contexto en el que se desenvuelve la economía. De acuerdo a Cuadrado \& Mancha (2003) los instrumentos de la política económica persiguen los siguientes objetivos:

- Tener la potestad para la reglamentación y la coacción sobre la actividad económica. 
- Capacidad para establecer la cantidad de dinero en circulación dentro de un país y los tipos de interés.

- Los instrumentos fiscales, los ingresos y los gastos públicos.

Figura 2.Instrumentos de la Política Económica

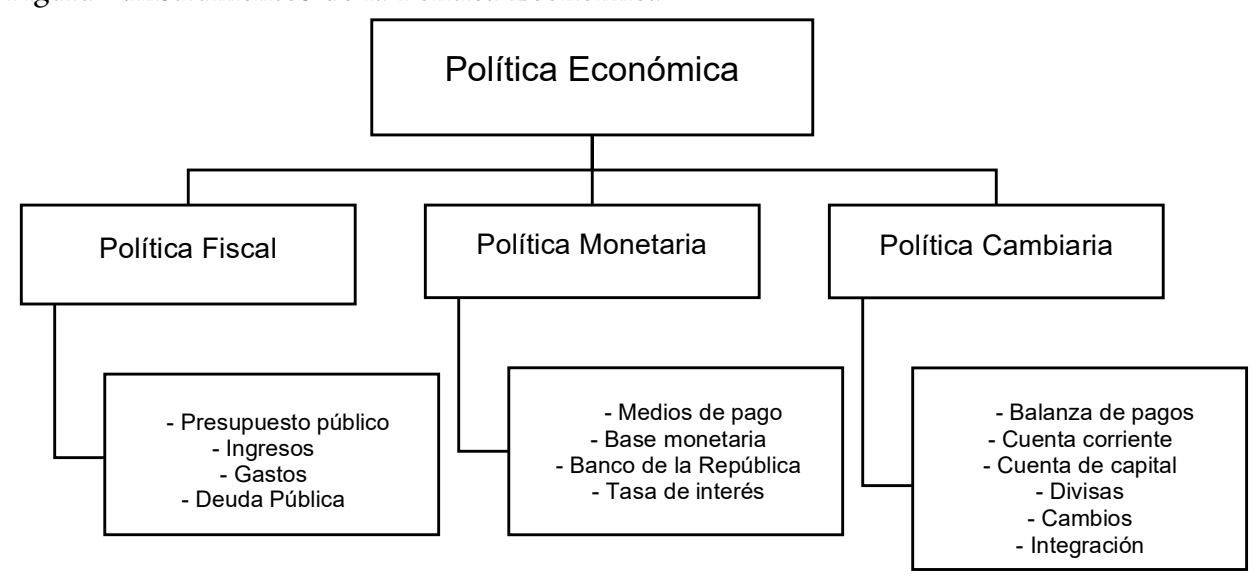

Nota: Política Económica, crecimiento y desarrollo humano: principales relaciones. Reyes (2008).

La política fiscal, monetaria y cambiaria es parte fundamental de la política económica de los países. Una adecuada coordinación de estos instrumentos contribuye a una asignación eficaz de los recursos dentro de una economía. Si su combinación ofrece estabilidad, se podría de cierta manera garantizar cierta sostenibilidad económica, beneficio social e incluso sustentabilidad ambiental en el desarrollo de la producción, distribución y consumo de bienes y servicios. Esto ocurre debido a que al existir capacidad de predicción sobre el comportamiento que presentan las variables económicas, los agentes económicos podrán contar con una base sobre la cual realizar planificaciones y desarrollar sus expectativas (Reyes, 2008).

\section{Metodología}

La metodología de la investigación responde a una revisión bibliográfica. De acuerdo a Peña (2010) la revisión bibliográfica en un texto cuyo propósito es presentar una síntesis de los textos analizados durante el proceso de investigación documental, para luego llevar a cabo la discusión de los hechos encontrados y las conclusiones.

Antes de iniciar la búsqueda de documentación es importante tener muy en claro el tema de investigación, lo que permitió iniciar la búsqueda de la información en diferentes fuentes como lo fueron: normas, reportes oficiales, revistas especializadas, memorias de conferencias, tesis doctorales, entre otros. El siguiente paso consistió en organizar la información. Se identificaron 182 documentos re- 
lacionados con el tema donde se dio prioridad a aquellos que exponían las ideas más importantes y los aspectos más relevantes para el tema de análisis. Esta acción se llevó a cabo a partir de la lectura de los resúmenes y las conclusiones de dichos documentos. Se identificaron 57 documentos de mayor interés para el estudio.

Estos documentos fueron leídos con mayor detalle y finalmente fueron elegidos 18 como realmente relevantes para la investigación, con los cuales se llevó a cabo la revisión bibliográfica. Siendo los aspectos que más resaltan de la investigación, lo que se expone a continuación.

En Ecuador, su historia se ha encontrada d eterminada por un pobre desempeño económico en el largo plazo. La economía del país se ha identificado por ser proveedora de materias primas y su dinamismo se ha encontrado caracterizado por una serie de booms cíclicos de exportación, principalmente de productos primarios, entre los que se encuentran: cacao (1866-1925); banano (1946-1968) y el petróleo (1972 - hasta la actualidad). Se puede afirmar que la economía ecuatoriana es relativamente pequeña, con marcada dependencia del comercio internacional y con un alto grado de inequidad (Banco Central del Ecuador, 2010). Entre los años 1972 y 2015, el Producto Interno Bruto (PIB) de Ecuador, que es el indicador utilizado con mayor frecuencia para medir la producción o el ingreso total en economía, ha mostrado un crecimiento con una tasa que se encuentra cercana al $4 \%$ anual. Pero al analizar los términos per cápita el PIB únicamente ha crecido a una tasa del 1,7\% anual, lo que hace que el Ecuador se encuentre visiblemente atrás con respecto a otros países de la región (Figura 3) (Díaz \& Ruiz, 2018).

Figura 3. PIB real per cápita de Ecuador y América Latina (1960-2014)

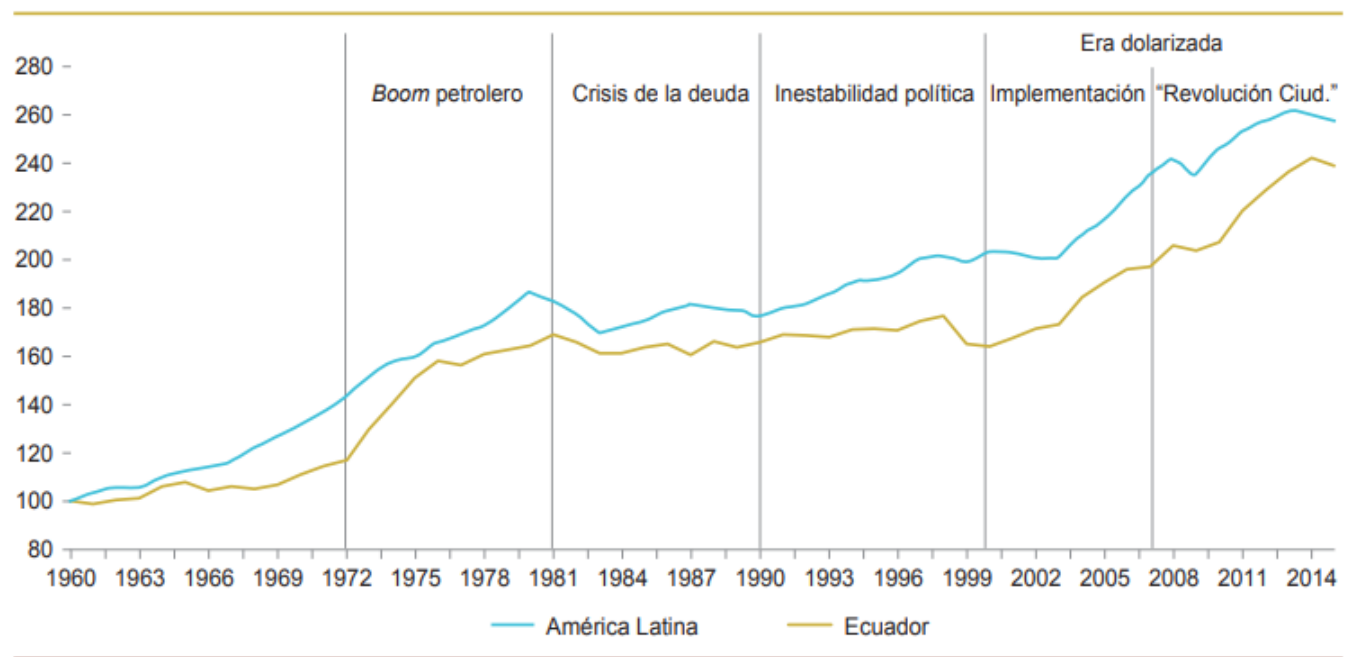

Nota: Banco Mundial (2019)

Las economías de América Latina, mantienen en general, mercados donde se observa que el nivel de competencia es menor al compararlo con el de otras regiones que se presentan más desarrolladas, esto puede verse reflejado en los altos márgenes de 
precios. Para Ecuador, la falta de competencia, y que también le impide alcanzar un mayor crecimiento económico, se estima que se encuentra asociada a las barreras de entrada como licencias y permisos, que al comportamiento que presentan los sistemas logísticos y la regulación antimonopolio (CAF, 2018). En la tabla 1 se presentan los principales indicadores macroeconómicos de Ecuador en el periodo $1972-2015$.

Tabla 1. Principales indicadores macroeconómicos, 1972-2015

\begin{tabular}{|c|c|c|c|c|c|c|c|}
\hline & Boom & Crisis & Inestabilidad & \multicolumn{3}{|c|}{ DOLARIZACIÓN } & \multirow{2}{*}{$\begin{array}{l}\text { Promedio } \\
1972-2015\end{array}$} \\
\hline & $\begin{array}{l}\text { petrolero } \\
(1972-81)\end{array}$ & $\begin{array}{l}\text { de la deuda } \\
(1982-89)\end{array}$ & $\begin{array}{l}\text { política } \\
(1990-99)\end{array}$ & $\begin{array}{l}\text { Promedio } \\
2000-15\end{array}$ & $\begin{array}{l}\text { Implementación } \\
(2000-06)\end{array}$ & $\begin{array}{l}\text { "Revolución } \\
\text { Ciudadana" } \\
(2007-15)\end{array}$ & \\
\hline PIB real & 6,9 & 2,1 & 2,3 & 4,1 & 4,3 & 3,9 & 4,0 \\
\hline $\begin{array}{l}\text { PIB per } \\
\text { cápita }\end{array}$ & 4,0 & $-0,4$ & 0,1 & 2,4 & 2,5 & 2,3 & 1,7 \\
\hline Inflación & 13,1 & 40,8 & 39,8 & 11,0 & 19,5 & 4,3 & 23,4 \\
\hline $\begin{array}{l}\text { Base } \\
\text { monetaria }\end{array}$ & 21,9 & 34,9 & 48,4 & 9,7 & 0,2 & 17,2 & 25,9 \\
\hline $\begin{array}{l}\text { Tipo } \\
\text { de cambio }\end{array}$ & 1,1 & 46,4 & 39,2 & - & - & - & 20,2 \\
\hline \multicolumn{8}{|c|}{ (porcentaje del PIB) } \\
\hline Balance fiscal & $-0,2$ & $-3,2$ & $-1,5$ & $-0,5$ & 1,4 & $-1,9$ & $-1,4$ \\
\hline $\begin{array}{l}\text { Balance } \\
\text { primario }\end{array}$ & 0,8 & 1,7 & 3,0 & 1,6 & 4,9 & $-1,0$ & 2,0 \\
\hline $\begin{array}{l}\text { Cuenta } \\
\text { corriente }\end{array}$ & $-3,3$ & $-3,8$ & $-2,4$ & 0,1 & 0,3 & 0,0 & $-1,9$ \\
\hline $\begin{array}{l}\text { Balanza } \\
\text { comercial }\end{array}$ & 0,9 & 4,1 & 2,7 & 0,7 & 1,4 & 0,2 & 1,8 \\
\hline $\begin{array}{l}\text { Deuda } \\
\text { externa }\end{array}$ & 12,4 & 55,8 & 61,5 & 25,0 & 37,4 & 15,3 & 36,0 \\
\hline \multicolumn{8}{|c|}{ (dólares por barril en dólares del año 2010) } \\
\hline $\begin{array}{l}\text { Precio del } \\
\text { petróleo } \\
\text { ecuatoriano }\end{array}$ & 39,85 & 34,23 & 22,08 & 57,85 & 38,16 & 73,16 & 41,33 \\
\hline $\begin{array}{l}\text { Precio del } \\
\text { petróleo WTI }\end{array}$ & 41,95 & 41,85 & 28,43 & 65,74 & 48,32 & 79,30 & 47,51 \\
\hline
\end{tabular}

Nota: La Deuda Pública, el crecimiento económico y la política. Periodo 1972 - 2015. (Álvarez, Álvarez, \& Álvarez, 2017)

Como se observa en la tabla 1, en el periodo del boom petrolero, donde la producción y comercialización de petróleo tomaron impulso, las condiciones del mercado internacional contribuyeron a que exista una notable mejora en lo que respecta a los términos de intercambio, debido al incremento del precio del petróleo en el mercado internacional (Cuesta, 2012). Luego del auge petrolero se observa perio- 
dos de crisis e inestabilidad, que desembocaron en la dolarización, hecho que se analizará más adelante. Para hacer frente a las crisis económicas que ha sufrido el país; marcadas por el déficit fiscal, la disminución del PIB y un deterioro general de las cuentas nacionales; los gobiernos han luchado frente a esta situación a partir de una política monetaria expansionista, lo que incluía una extensión del financiamiento hipotecario, y de acuerdo a la escasa política monetaria aplicada, se trataban de mantener las tasas de interés bajas, de esta manera se limitaban las reservas bancarias que podrían salir del país. Se trató de estimular la economía, no solo a través del crédito hipotecario, sino también se consideró un aumento sobre el Crédito de Desarrollo Humano.

A partir de la implementación de diversas políticas económicas y sociales, se logró que para el año 2009 la pobreza haya disminuido en aproximadamente el 17\%. Se estima que la reducción de la pobreza ha encontrado apoyo en la ampliación del Bono de Desarrollo Humano, el cual es un programa de transferencias monetarias hacia personas de bajos ingresos, destinados principalmente a madres solteras con hijos menores de edad, adultos mayores de 65 años o personas que tengan alguna discapacidad. El gobierno nacional afirma que son diversos los estudios realizados donde se certifica que hay efectos positivos y significativos para la salud y la educación, que se encuentran directamente relacionados con el Bono de Desarrollo Humano.

En cuanto al aspecto económico relacionado con su balanza de pagos, se estima que Ecuador podría mejorar su situación si existiera una mayor diversificación en las exportaciones, debido a que pocos sectores, exceptuando al petróleo se han diversificado, pero la situación monetaria de Ecuador (dolarización) se presenta como una dificultad para lograr este objetivo (Ministerio de Relaciones Exteriores y Movilidad Humana, s.f.). Desde este punto es importante resaltar el papel de la política monetaria en la estabilidad económica y crecimiento de los países, el cual es un aspecto que ha sido ampliamente estudiado en los últimos años, si bien es cierto, son mayoritarios los estudios destinados a economías desarrolladas, también es necesario destacar las situaciones que atraviesan los países en desarrollo, especialmente aquellos que se encuentran ubicados en América Latina.

Antes de explicar la situación por la que atravesó el Ecuador y que incluyó la renuncia a su soberanía monetaria, es importante explicar cómo ciertos países se han visto en la necesidad de renunciar a sus monedas y adoptar a otra extranjera, principalmente lo hicieron como medida para superar sus problemas económicos. Es importante acotar que los países que deciden adoptar una moneda extranjera como su patrón monetario, lamentablemente pierden su capacidad de acción frente a posibles desequilibrios fiscales, estos problemas pueden darse por la falta de liquidez, además de que a nivel internacional pierde competitividad frente a aquellos países que se encuentran en la capacidad de devaluar su moneda, lo que les permite ser más competitivos al establecer mejores precios a su producción (Giler, Moretti, Mayor, León \& González, 2018). 
A inicios del siglo XXI Ecuador tomó la decisión de adoptar la dolarización con el propósito de afrontar su mayor crisis financiera de la historia. La adopción del dólar se llevó a cabo en el gobierno de Jamil Mahuad, en enero del 2000, siendo una de las decisiones de mayor impacto en la economía ecuatoriana, desatando un gran debate sobre los mecanismos de ajuste y validez utilizados para llevar a cabo el proceso de dolarización. La dolarización en Ecuador tiene una particularidad, el Banco Central aún tiene sus funciones de regulador de la actividad monetaria, aunque la mayor parte de la política monetaria opera a través de una variable instrumental, la Reserva Internacional de Libre Disponibilidad (RILD), por medio de su uso independiente, posibilitando de esta manera la regulación de la cantidad de dinero disponible en la economía (Carrasco, 2013).

Entre las razones por las que el gobierno de ese entonces decidió adoptar la dolarización, fue la pérdida de confianza en el sucre (moneda de Ecuador), además de la grave crisis financiera, lo que condujo a la moneda a un proceso de devaluación. La falta de credibilidad del Banco Central, máxima autoridad monetaria, sumado a la devaluación incontrolable de la moneda, contribuyeron a que las personas se refugien en una moneda más fuerte, el dólar, llegando la situación a ser insostenible (Ordeñana, 2011). Las cifras que anteceden a la dolarización, dan cuenta de un estancamiento económico y de escasa apertura comercial, con graves problemas estructurales de inequidad social, exclusión y pobreza. Para el año 1995 la pobreza había alcanzado al 56\% de la población, mientras que en el área rural llegaba a cifras que la ubicaban en el $76 \%$, cifras que son sumamente superiores a la media de la región.

La concentración del ingreso, calculada por el coeficiente de Gini de 0,57 ubicaba a Ecuador en una posición inferior al resto de la región (Banco Interamericano de Desarrollo, s.f.). En el aspecto social, el 57\% de la población urbana realizaba sus actividades económicas desde puestos de baja productividad, el analfabetismo estaba presente en el 10,5\% de la población, mientras que la escolaridad de la población adulta apenas completaba los 7 años. La salud también pasaba por un periodo crítico, en el año de1998, el 26\% de los niños menores de 5 años tenían desnutrición crónica (Larrea, 2004). Las cifras mencionadas, la inestabilidad política, el alto grado de corrupción, las relaciones conflictivas con el Perú, el gran déficit fiscal y la mala práctica de ciertas instituciones financieras, resultaron determinantes para la crisis económica que presentaba Ecuador. Esta crisis también se trasladó al sector público donde el déficit fiscal se ubicó en el $-12 \%$ del PIB. Al tener un mayor gasto público, Ecuador tuvo que dejar de pagar su deuda externa, perjudicando su acceso a créditos internacionales. La inflación se ubicó también en un porcentaje insostenible.

Se estima que el impacto de la dolarización en la economía ecuatoriana es, en términos generales, positivo, estos se debe al hecho de que ha brindado estabilidad económica al país, permitiendo a las personas tomar decisiones al largo plazo, una 
señal inequívoca de esta situación es el despegue del sector inmobiliario en ciudades como Guayaquil, Quito y Machala, donde se han llevado a cabo proyectos de viviendas y la construcción de urbanizaciones, todo esto debido a la capacidad de endeudamiento que poseen ahora las personas, situación que antes de la dolarización era prácticamente imposible. Para algunos analistas económicos, el peligro para la dolarización reside en la capacidad de reacción que tenga el país ante un shock externo, situación por la que Ordeñana (2011) afirma que estos riesgos se encuentran en constantes análisis. Volviendo al análisis de los efectos de la dolarización, se observó que su mayor efecto al corto plazo fue la reducción de la inflación.

Debido al sistema dolarizado que tiene Ecuador, es posible controlar la inflación y generar estabilidad macroeconómica, se considera que la dolarización ofrece un contexto más sano para la asignación de los recursos por parte del sector financiero. Ayudó a mejorar la estabilidad financiera debido a que las instituciones financieras tienen un mayor acceso a los fondos internacionales, así mismo el portafolio de dichas instituciones se considera que se volvió más sano, debido a que los activos y pasivos, tanto de las instituciones financieras como los de sus clientes, encontraron equilibrio en términos de moneda.

Sin embargo, a pesar de los aspectos positivos mencionados, la dolarización también implica aspectos negativos. Uno de los principales, es el haber eliminado la posibilidad de que el Banco Central del Ecuador desempeñe el rol de prestamista de última instancia en el caso de que suceda una corrida bancaria. Por lo tanto, actualmente, uno de los pilares de decisiones de política económica en Ecuador, y que podría influir en el crecimiento, es la aplicación de una política fiscal, la misma que debe validar el aumento del gasto público, el incremento de la recaudación tributaria, las transferencias, especialmente aquellas destinadas al desarrollo de programas sociales y los subsidios (Arévalo, 2014).

Dentro del sistema de la dolarización, la política monetaria no existe, lo que le impide al gobierno disponer de medidas de política cambiaria que le permita protegerse de los shocks externos. La economía ecuatoriana en los últimos años se ha mostrado vulnerable a los shocks externos, lo cual es consecuencia de su apertura a los mercados internacionales, aunque también se debe a la gran dependencia de la economía del sector fiscal y de los recursos provenientes de la venta de petróleo. Dentro de la Política Fiscal ecuatoriana, el Presupuesto General del Estado se ha llegado a configurar como una variable de control, cuyo propósito es el de asegurar y mantener la estabilidad económica y, al mismo tiempo la inflación y el desempleo. Desde el presupuesto del Estado se realiza una especial atención al papel que desempeña el gasto público y analiza sus efectos jurídicos, económicos y financieros (Giménez, 2013)

Al realizar un análisis del presupuesto del Estado (Figura 4), en el periodo 2009 - 2018 se observa como el Presupuesto General del Estado de Ecuador se ha ido incrementando de forma paulatina, esta situación es consecuencia del elevado gasto público del gobierno del Econ. Rafael Correa, tanto en el año 2016 como en 
el 2018 se observa una disminución, la primera asociada a la crisis económica producto de la disminución del precio del petróleo y la segunda se debe a los intentos del gobierno de Lenin Moreno por reducir el déficit fiscal.

Figura 4. Evolución del Presupuesto General del Estado Ecuador. Periodo 2009-2018.

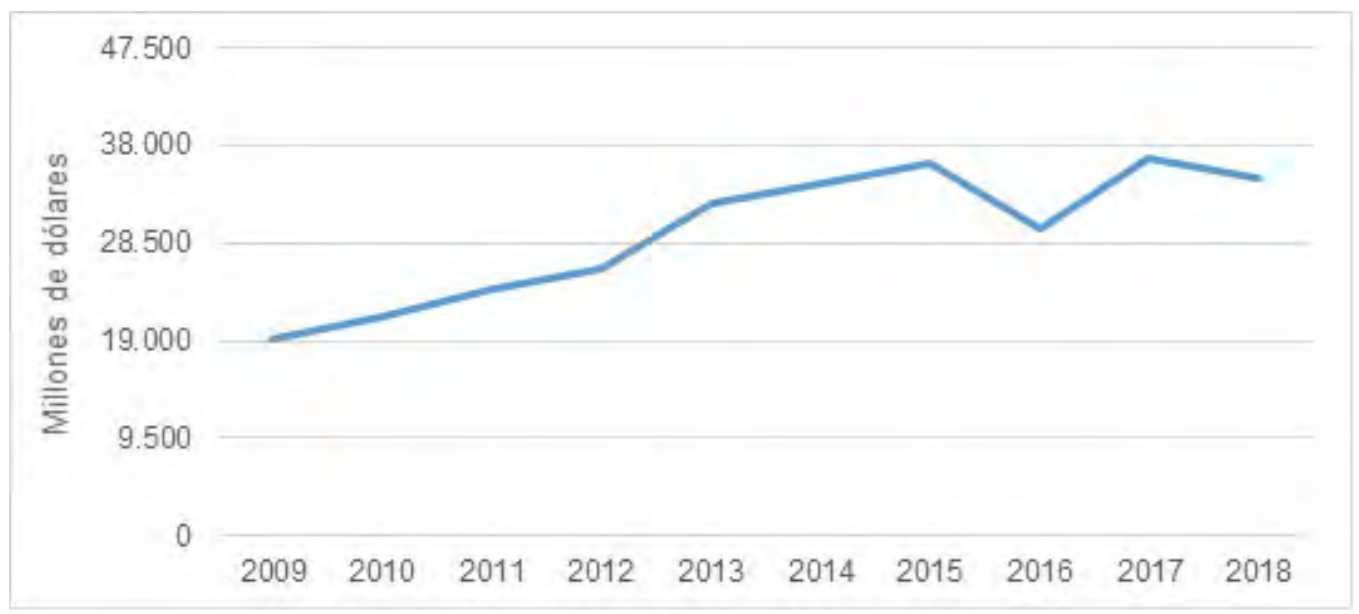

Nota: Programa Económico (Ministerio de Economía y Finanzas, 2018)

El presupuesto público es una muestra de cómo el Gasto Público se ha venido incrementando de forma sostenida, hecho que se expone en la Figura 5. El manejo del gobierno ecuatoriano en los últimos años, dan cuenta de cómo el Estado ha estado imponiendo políticas económicas sustentadas en un fuerte gasto público, debido a que como Estado no puede disponer de una política cambiaria, el propósito principal ha sido el de armar una estructura donde los recursos ingresen de forma permanente, de esta manera la política tributaria se ha convertido en una herramienta que permite asegurar los fondos presupuestarios.

Figura 5.Evolución del Gasto Público. Periodo 2007 - 2017.

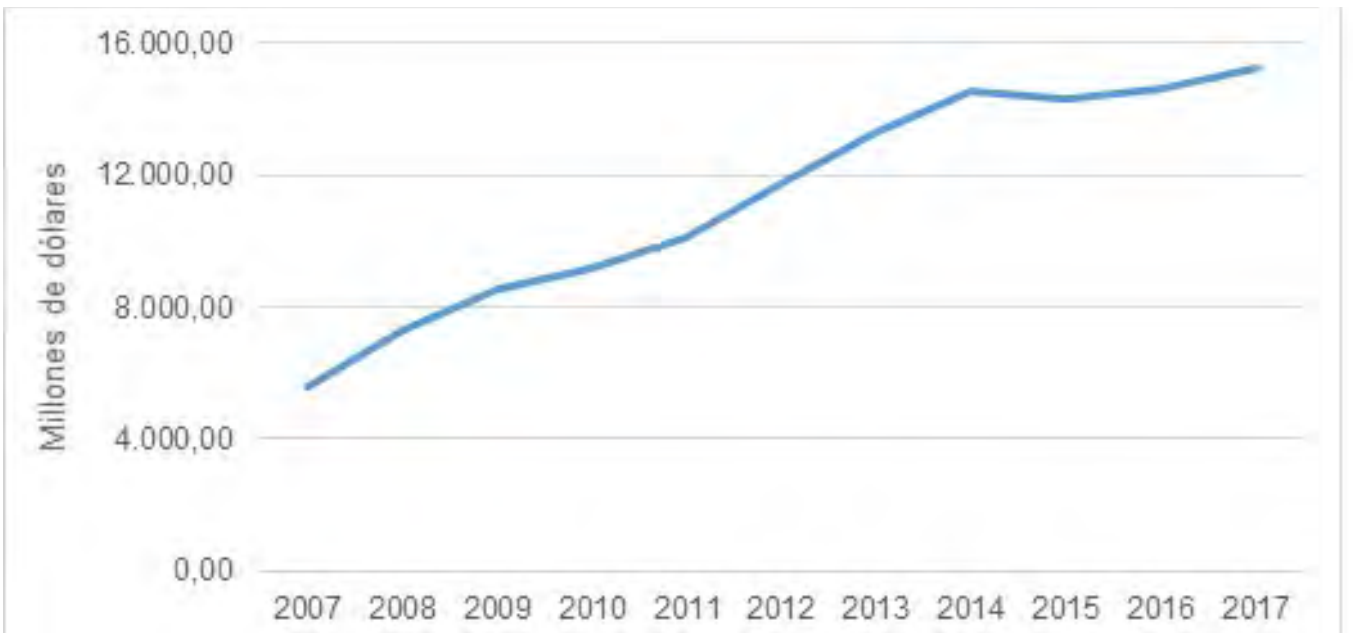

Nota: Programa Económico (Ministerio de Economía y Finanzas, 2018) 
El Gasto Público se ha incrementado de forma sostenida desde el año 2007 ocasionando un gran déficit fiscal, ante esta situación el gobierno de Lenin Moreno ha implementado diversas medidas, entre las que se encuentran la eliminación de instituciones, la reducción de salario, el despido de servidores públicos, entre otros. El gasto se ha convertido en uno de los temas de mayor debate en cuanto al crecimiento y desarrollo económico, especialmente al momento de establecer la relación entre el gasto del gobierno y el desarrollo económico.

Desde este contexto se afirma que el gasto del gobierno tiene un efecto significativo y positivo sobre el incremento del PIB y, por lo tanto, sobre el crecimiento económico (Campo \& Mendoza, 2018). Para Molina \& Gantier (2017) el análisis del gasto público es tema de gran importancia, debido a que a través de este se dinamiza la economía lo que contribuye al crecimiento económico y a la ejecución de políticas económicas y sociales que conduzcan al bienestar de la población. A través del gasto público se puede asignar de forma eficiente sus recursos y proveer de los servicios públicos a la población (Ibarra, 2010).

Para que los gobiernos puedan solventar su gasto público es necesario tener ingresos, en este sentido la recaudación tributaria es de gran importancia. Los ingresos tributarios son aquellos que se originan en la potestad que posee el Estado para el establecimiento de gravámenes, como son los diversos impuestos. Estos recursos les permitirán desarrollar las actividades que fueron programadas para el sector público, atender las obligaciones de pago de la deuda pública o llevar a cabo las transferencias que requieran otros ámbitos a nivel gubernamental (Almeida, 2015).

En Ecuador, los ingresos obtenidos a través de la recaudación tributaria son la principal fuente de financiamiento del gobierno. Como se evidencia en la Figura 6, durante el periodo 2007 - 2017 la recaudación tributaria se ha ido incrementando paulatinamente como consecuencia de las medidas implementadas para evitar la evasión y elusión fiscal. El año de mayor recaudación fue en el 2015 con una pequeña disminución para el año 2016 y 2017. Con respecto a los impuestos, los de mayor recaudación tributaria son: el Impuesto al Valor Agregado (IVA) y el Impuesto a la Renta (IR); luego de estos se encuentran el Impuesto a los Consumos Especiales (ICE) y el Impuesto de Salida de Divisas (ISD).

Es necesario mencionar que cada país tiene su legislación que le permitirá llevar a cabo la recaudación, por lo tanto, las reformas fiscales se adaptan a los contextos particulares que presenta cada país, y donde son considerados aspectos como: cultura, historia, política, geografía, los mismos que tienen efecto sobre cualquier política (Casares, García, Ruíz, \& Sobarzo, 2015).

Otra variable de análisis y que incide sobre el crecimiento económico de los países es la deuda externa. De acuerdo a la teoría económica, un nivel razonable de endeudamiento facilita el crecimiento y el desarrollo económico de los países (Pattillo, Poirson, \& Ricci, 2002). Siempre y cuando los préstamos sean destinados para inversiones productivas y no se encuentre afectado por la inestabilidad ma- 
croeconómica, ni políticas que distorsionen los incentivos, deberían experimentar crecimiento y de encontrarse en la capacidad de realizar reembolsos puntuales. Pero cuando los índices de endeudamiento alcanzan niveles muy altos, queda claro que los países no podrán realizar los reembolsos, lo que afectará el desempeño económico y, por lo tanto, no se alcanzará el crecimiento.

Figura 6.Evolución de la Recaudación Fiscal. Periodo 2007 - 2017

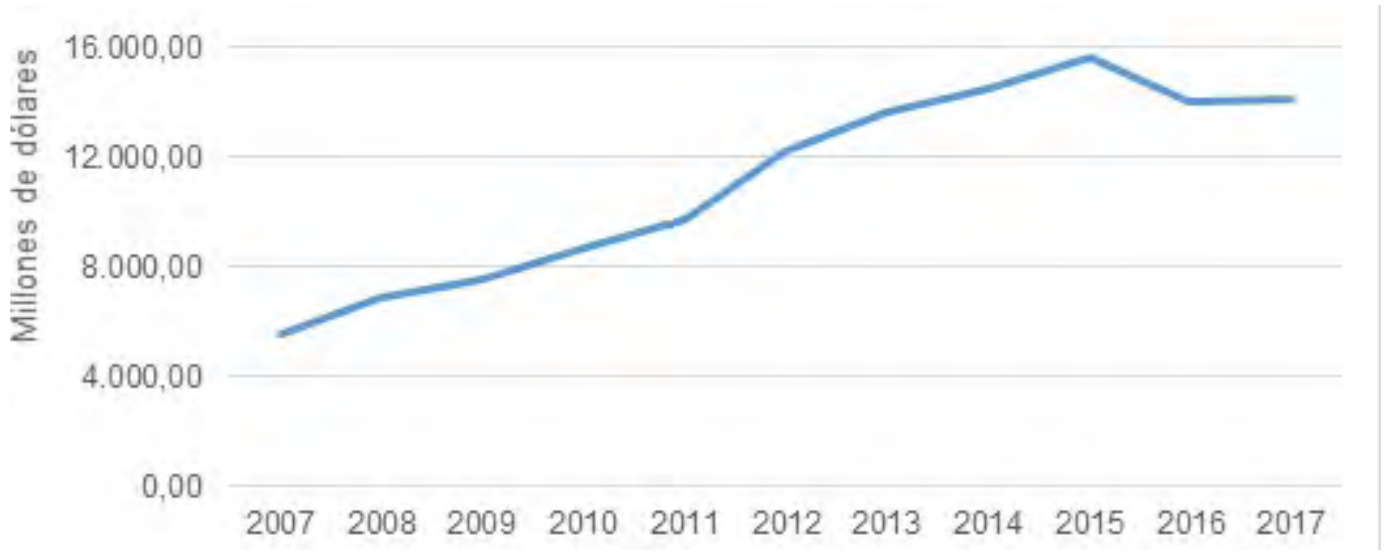

Nota: Programa Económico (Ministerio de Economía y Finanzas, 2018)

En Ecuador, la deuda externa ha sido motivo de gran preocupación desde la independencia, los malos manejos y asignaciones de los préstamos e incluso los actos de corrupción contribuyeron a que la deuda aumente hasta cifras insostenibles. A finales del 2006 la deuda se ubicaba en los 10.000 millones de dólares americanos, durante el periodo presidencial de Rafael Correa se anuncia el no pago de la deuda al considerarla ilegitima (Lucio, 2019).

En la actualidad China se mantiene como el principal socio financista de Ecuador, donde han sido entregados varios préstamos que, según datos del gobierno nacional, han sido invertidos en varios proyectos estratégicos y también han ingresado como pago de la venta anticipada de petróleo. Aproximadamente 6.000 millones de dólares americanos son la deuda de Ecuador con China, deuda que se incrementó drásticamente a partir del año 2015 (Figura 7) (Álvarez, Álvarez, \& Álvarez, 2017).

Con respecto a la deuda interna, esta se aproxima a los 14 mil millones de dólares americanos. Los principales acreedores del gobierno son el Banco Central del Ecuador (BCE) y el Instituto Ecuatoriano de Seguridad Social (IESS). La deuda interna es una de las principales preocupaciones del gobierno ecuatoriano, esta deuda encierra el compromiso del gobierno para ofrecer bienestar a sus ciudadanos. Su evolución se muestra en la Figura 7.

Las exportaciones también son un aspecto de gran importancia en el crecimiento económico. Autores como Kaldor (1976), Kalecki (1977) y Thirlwall (2003), sugieren que el comercio internacional, y, sobre todo, las exportaciones, es la lo- 
comotora del crecimiento económico (Gaviria, s.f.). Las principales exportaciones de Ecuador son: petróleo crudo, plátanos, crustáceos, pescado procesado y flores cortadas. Las cifras de las exportaciones llegan al 18\% del total del Producto Interno Bruto (PIB), el 12\% corresponde a exportaciones no petroleras y un $6 \%$ a exportaciones petroleras. Del porcentaje de exportaciones no petroleras, tan solo un $2 \%$ pertenece a exportación de servicios. En la Figura 8 se presentan la evolución de las exportaciones.

Figura 7.Evolución de la Deuda Externa y Deuda Interna

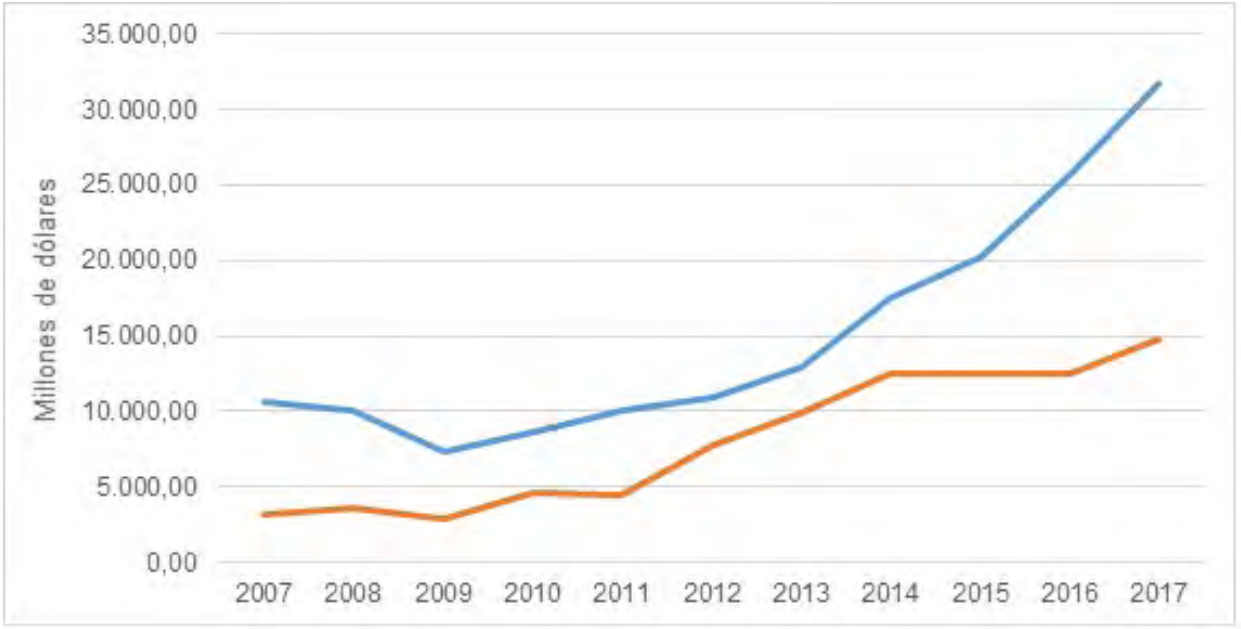

Nota: Programa Económico (Ministerio de Economía y Finanzas, 2018)

Figura 8. Evolución de las Exportaciones de Ecuador. Periodo 2007 - 2017

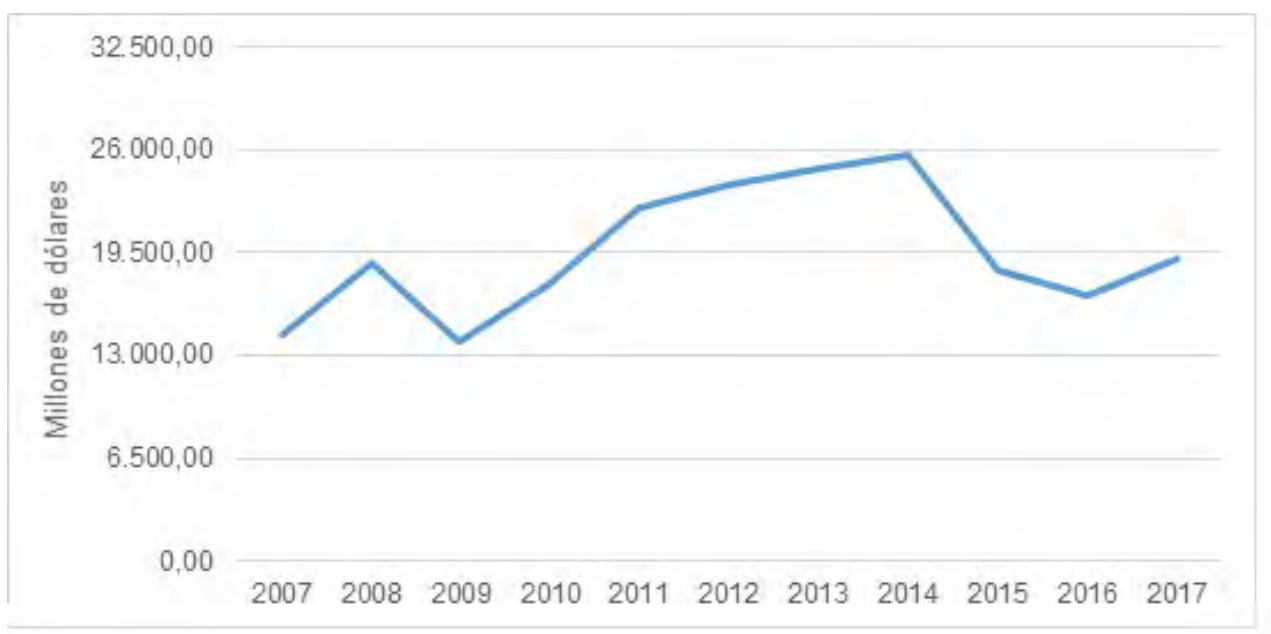

Nota: La Deuda Pública, el crecimiento económico y la política. (Álvarez, Álvarez, \& Álvarez, 2017)

Las importaciones pueden incidir en el comportamiento del PIB de una nación en dos vías: primero, en el corto plazo pueden resultar lesivas para el crecimiento del PIB, porque al aumentar las importaciones se perjudica o lesiona la demanda externa. En segundo lugar, a largo plazo las importaciones pueden jugar un papel 
fundamental en el crecimiento económico de los países, pues estas generalmente no se hacen por bienes finales sino por bienes intermedios, siendo por tanto relevante los tipos de bienes importados y no el valor de su monto anual (Gómez \& Ramírez, 2017).

Figura 9. Evolución de las importaciones. Periodo 2007 - 2017.

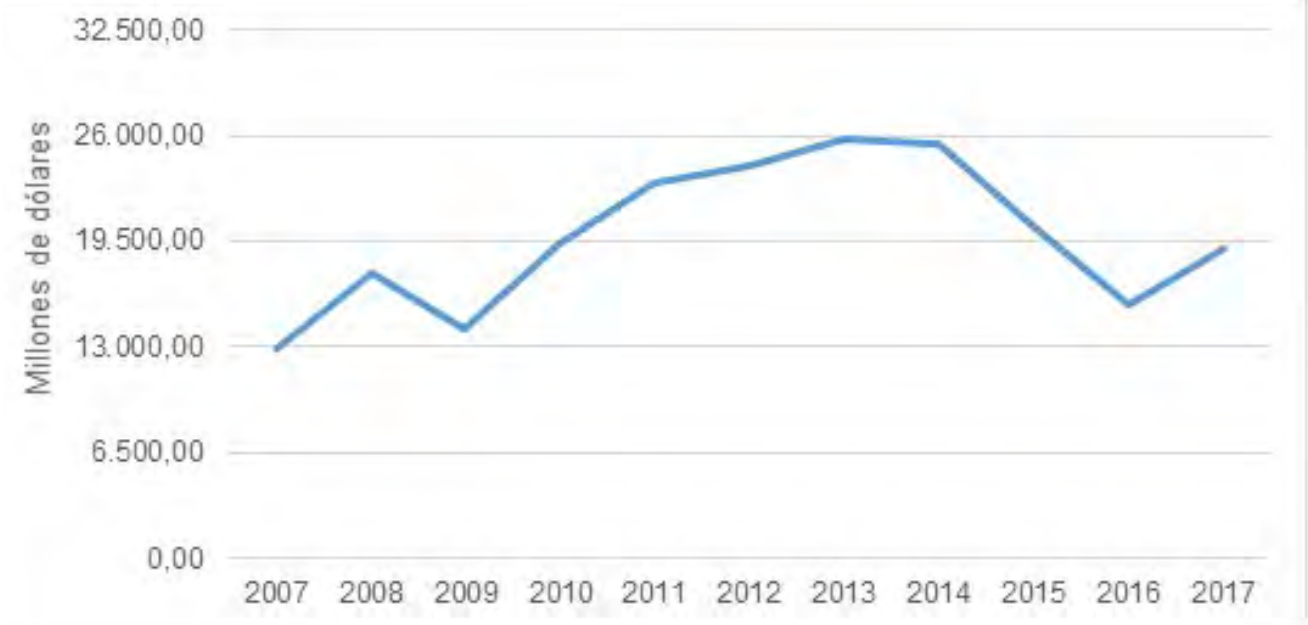

Nota: La Deuda Pública, el crecimiento económico y la política. (Álvarez, Álvarez, \& Álvarez, 2017)

Las principales importaciones de Ecuador son: refinado de Petróleo, alquitrán de aceite, medicamentos envasados, coches y trigo. El punto máximo de las importaciones se encuentra en el año 2014. Las compras al exterior representan el 20,01\% del PIB, al observar la figura 9 se evidencia la evolución de las importaciones en Ecuador en los últimos años con respecto al año 2007, con una disminución en el año 2016 y con una leve recuperación en el año 2017.

Una de las consecuencias del excesivo gasto público de Ecuador es el déficit fiscal, el cual se ha mantenido progresivamente a lo largo de los años. Muchos analistas económicos sugieren que mantener el déficit público estable y bajo nivel de deuda interna y externa, contribuiría a estimular la inversión extranjera directa, pública y privada, contribuyendo al desarrollo y crecimiento económico del país.

\section{Conclusiones}

La economía al nivel del ámbito mundial ha llegado a enfrentar diversos cambios en los últimos años, estos cambios se relacionan con el progreso tecnológico, lo que redujo las distancias y costos de producción modificando las formas tradicionales de organización laboral, circulación y el consumo de bienes y servicios. Los cambios mencionados también se relacionan con la transformación social y la garantía de mejores equilibrios a través de la Política Social, ocasionando modificaciones en 
las funciones del Estado Nacional, con respecto a la dirección que tendrá la Política Económica.

Antes de la dolarización los gobiernos no lograban definir una política económica que contribuya a la transformación de los rasgos estructurales de tipo rentista que caracterizaba a la relación del Estado con los actores de la economía. La crisis del año 1999 puso en evidencia que esta situación ya era insostenible sumado a la gran corrupción en el uso de los recursos públicos, la única salida que encontró el gobierno de Jamil Mahuad es la instauración de la dolarización en la economía ecuatoriana. Una vez implementada la dolarización se ha obtenido cierta estabilidad económica que se ve reflejada en los indicadores económicos y sociales. Aunque si bien el principal problema al que se enfrenta el gobierno ecuatoriano es el hecho de que el único mecanismo para la intervención de la economía es la política fiscal, lo que es restrictivo en cuanto a la aplicación de la política monetaria.

En el Ecuador debido a la ausencia de política monetaria, es la política fiscal la que permite contrarrestar las fluctuaciones de corto plazo en la actividad económica, siendo también una herramienta de gran valor para influir sobre el nivel de producción potencial de la economía por medio de la inversión pública en capital físico como humano. Por lo tanto, los pilares del crecimiento económico de Ecuador a partir del año 2000, fueron la aplicación de una política fiscal, que validaba el aumento del gasto público, el incremento de la recaudación tributaria, las transferencias sociales. Esto ha promovido el déficit fiscal, es decir al gasto excesivo de los recursos del Estado. 


\section{Referencias}

Almeida, P. (4 de Agosto de 2015). Recaudación y Gasto Público. Obtenido de Observatorio Económico Social UNR: http://www.observatorio.unr.edu.ar/recaudacion-y-gasto-publico/

Álvarez, M., Álvarez, M., \& Álvarez, S. (2017). La Deuda Pública, el crecimiento económico y la política. Polis: Investigación y Análisis Sociopolitico y Psicosocial, 13(2), 41-71. Obtenido de https://www.redalyc.org/pdf/726/72654751003.pdf

Arévalo, G. (2014). Ecuador: economía y política de la revolución ciudadana, evaluación preliminar. Revista Apuntes del CENES, 33(58), 109-134. Obtenido de https://www.redalyc.org/pdf/4795/479547210005.pdf

Banco Central del Ecuador. (2010). La economía ecuatoriana, luego de 10 años de dolarización. Quito: BCE.

Banco Interamericano de Desarrollo. (s.f.). Perspectiva general. IDB.

Cachanosky, I. (31 de Octubre de 2012). Economía, en la teoría y en la política. Obtenido de elcato.org: https://www.elcato.org/economia-en-la-teoria-y-en-lapolitica

CAF. (13 de Noviembre de 2018). La baja productividad, asignatura pendiente de Ecuador y del resto de América Latina. Obtenido de https://www.caf.com/es/actua$\underline{\text { lidad/noticias/2018/11/la-baja-productividad-asignatura-pendiente-de-ecua- }}$ dor-y-del-resto-de-america-latina/

Campo, J., \& Mendoza, H. (2018). Gasto público y crecimiento económico: un análisis regional para Colombia, 1984-2012. Lecturas de Economía(88), 77-108. Obtenido de http://www.scielo.org.co/pdf/le/n88/0120-2596-le-88-00077.pdf

Carrasco, A. (2013). La politica monetaria en el Ecuador. Quito: Servicios de Rentas Internas.

Carrillo, P. (2017). El efecto de la política fiscal en expasión y recesión para Ecuador: Un modelo MSVAR. Cuadernos de Economía, 36(71), 405-439.

Casares, E., García, M., Ruíz, L., \& Sobarzo, H. (2015). Distribución del ingreso, impuestos y transferencias en México. Un análisis de equilibrio general aplicado. El Trimestre Económico, 82(3), 523-558. Obtenido de https://www.redalyc. org/pdf/313/31342334002.pdf

Cuesta, P. (2012). Análisis del Impacto de la Deuda Externa sobre el Producto Interno Bruto (PIB) en el Ecuador, Período 1970 - 2010. Cuenca: Universidad de Cuenca.

Díaz, J., \& Ruiz, M. (2018). Reformas y Desarrollo en el Ecuador contemporáneo. Qui- 
to: Banco Interamericano de Desarrollo.

García, J., \& Zabala, H. (2008). Políticas de Estado como sustento de la organización ciudadana. CICAG. Centro de Investigación de Ciencias Administrativas y Gerenciales, 5(2), 111-129.

Gaviria, M. (s.f.). Exportaciones y Crecimiento Económico. Colombia: Universidad Católica de Pereira.

Giler, J., Moretti, A., Mayor, M., León, M., \& González, J. (2018). Efectos de no poseer una política monetaria y su incidencia en la competitividad del Ecuador. Observatorio de la economía latinoamericana(1696-8352). Obtenido de https:// www.eumed.net/rev/oel/2018/03/competitividad-ecuador.html

Giménez, M. (2013). Presupuesto, gasto público y acceso a la información. Aspectos relevantes del decreto $\mathrm{N}^{\circ}$ 692/09 del Poder Ejecutivo de la Provincia de Santa Fe en materia de Finanzas Públicas. Documentos y Aportes en Administración Pública y Gestión Estatal, 13(20), 207-210. Obtenido de http://www.redalyc. org/pdf/3375/337530224009.pdf

Gómez, A., \& Ramírez, Z. (2017). Causalidad entre las importaciones y el crecimiento económico: evidencia empírica para el departamento del Cauca (Colombia). Revista Facultad de Ciencias Económicas, 25(2), 41-62. Obtenido de http://www.scielo.org.co/pdf/rfce/v25n2/0121-6805-rfce-25-02-00041.pdf

Gutiérrez, O. (2009). Sobre la política económica. Perspectivas (24), 37-58. Obtenido de http://www.redalyc.org/pdf/4259/425942160003.pdf

Hunt, S. (2007). Economic growth: should policy focus on investment or dynamic competition? European Business Review, 19(4), 274-291.

Ibarra, A. (2010). Concepto de Gasto Público. México: EUMED.

Larrea, C. (2004). Crisis, dolarización y pobreza en el Ecuador. Quito: Editoria Abya-Yala.

Lucio, P. (2 de Febrero de 2019). Historia de deuda externa. El Universo, págs. https://www.eluniverso.com/opinion/2019/02/02/nota/7168730/historia-deuda-externa.

Ministerio de Relaciones Exteriores y Movilidad Humana. (s.f.). Ecuador con resultados positivos en su Economía. Obtenido de https://www.cancilleria.gob.ec/ ecuador-con-resultados-positivos-en-su-economia/

Molina, G., \& Gantier, M. (2017). El Gasto Público y su Efecto en la Economía Boliviana: Periodo 1990-2015. PERSPECTIVAS, 20(39), 7-42. Obtenido de https://www.redalyc.org/pdf/4259/425951181002.pdf

Ontaneda, D. (2017). El impacto de la dolarización oficial en la profundización 
financiera en Ecuador. Cuestiones Económicas, 27(1), 13-43. Obtenido de https://www.bce.fin.ec/cuestiones economicas/images/PDFS/2017/No1/El\%20 impacto\%20de\%20la\%20dolarizaci\%C3\%B3n\%20oficial\%20en\%20la\%20profundizaci\%C3\%B3n\%20financiera\%20en\%20Ecuador.pdf

Ordeñana, X. (2011). El impacto de la dolarización en la economía ecuatoriana, once años después de su implementación. Retos, 2(1), 165-171.

Pattillo, C., Poirson, H., \& Ricci, L. (2002). La deuda externa y el crecimiento. Finanzas \& Desarrollo, 32-35. Obtenido de https://www.imf.org/external/pubs/ft/ fandd/spa/2002/06/pdf/pattillo.pdf

Peña, L. (2010). La revisión bibliográfica. Bogotá: Pontificia Universidad Javeriana.

Reyes, G. (2008). Política Económica, crecimiento y desarrollo humano: principales relaciones. Revista Tendencias, 9(1), 101-126.

Rodríguez, M., \& Rodríguez, E. (2009). Política económica y política social, como una política pública para combatir la pobreza. Espacios Públicos, 12(25), 123150. Obtenido de http://www.redalyc.org/articulo.oa?id=67611350008

Torres, F., \& Rojas, A. (2015). Política Económica y Política Social en México: desequilibrio y saldos. Problemas del Desarrollo. Revista Latinoamericana de Economía, 46(182), 41-65. Obtenido de https://www.redalyc.org/pdf/118/11840300003. pdf 
Universidad Técnica de Machala

Ediciones UTMACH

Primera edición en español 2020

PDF interactivo 


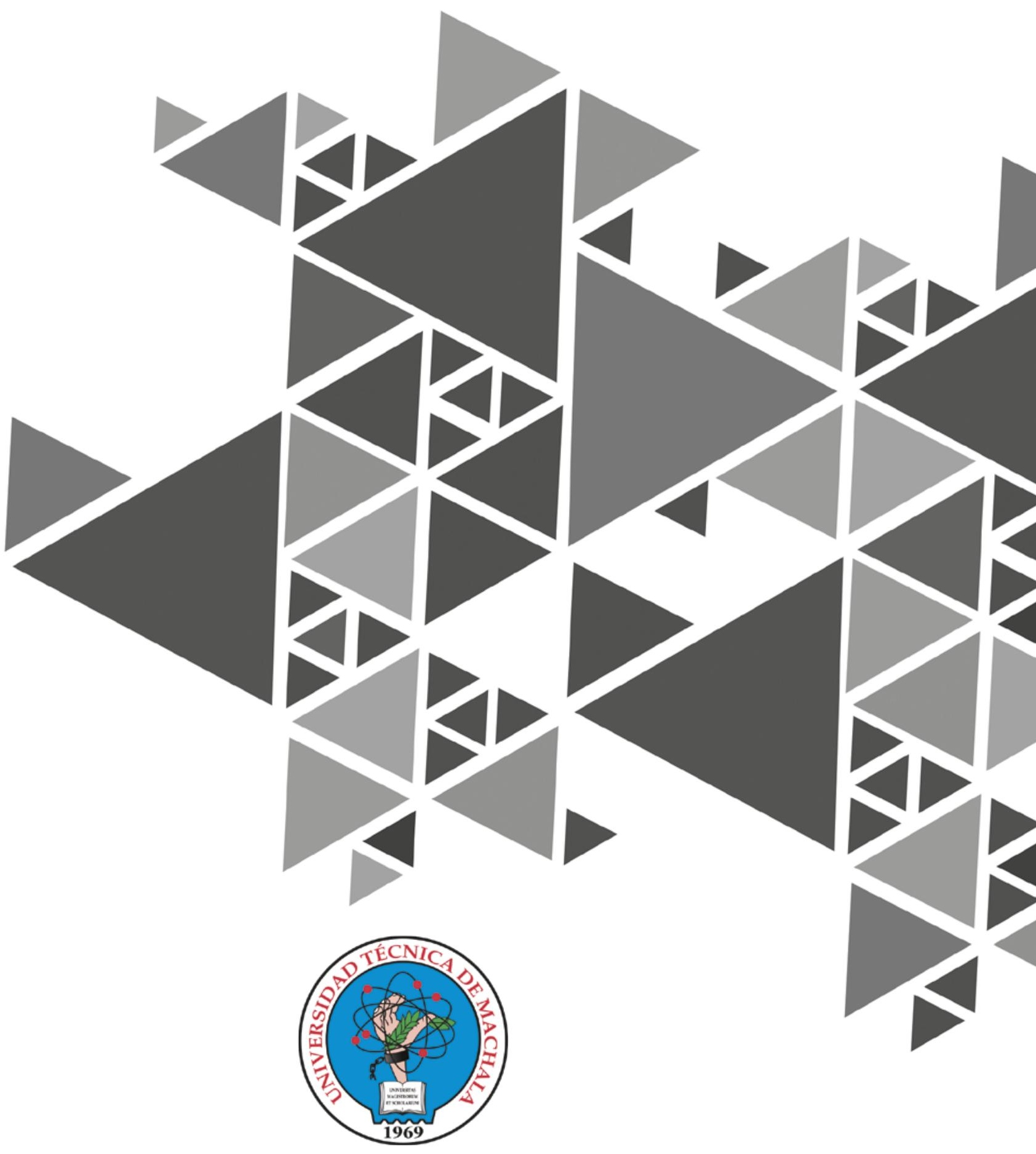

Universidad Técnica de Machala

Dirección de Investigación 